\title{
Photonic excess noise and wave localization
}

\author{
C W J Beenakker and M Patıa \\ Instituut Lorentz, Unversiteit Leiden, PO Box 95062300 RA Letden The Netherlands \\ P W Brouwer \\ Laboratory of Atomic and Solid State Physics, Cornell University, Ithaca New York, 14853 \\ (Recelved 12 January 2000, published 30 March 2000)
}

\begin{abstract}
This is a theory for the effect of localization on the super-Poissonian noise of radiation propagating through an absorbing disordered waveguide Localızation suppresses both the mean photon current $\bar{I}$ and the noise power $P$, but the Fano factor $P / \bar{I}$ is found to remain unaffected For strong absorption the Fano factor has the universal value $1+\frac{3}{2} f$ (with $f$ the Bose-Enstein function), regardless of whether the waveguide is long or short compared to the localization length
\end{abstract}

PACS number(s) $4250 \mathrm{Ar}, 4225 \mathrm{Dd}$

The coherent radiation from a laser has Poisson statistics $[1,2]$ Its noise powel $P_{\text {Poisson }}$ equals the mean current $\bar{I}$ (in units of photons per second) Elastic scattering has no effect on the noise, because the radiation remains in a coherent state The coherent state is degraded by absorption, resulting in an excess noise $P-P_{\text {Poisson }}>0$ [3] The Fano factor $P / P_{\text {Poisson }}$ deviates from unity by an amount proportional to the Bose-Einstein function $f$ It is a small effect $\left(f \sim 10^{-2}\right.$ at room temperature for infrared frequencies), but of interest because of its fundamental origin The excess noise is required to preserve the canonical commutation relations of the electromagnetic field in an absorbing dielectric [4-6]

The interference of multiply scattered waves may lead to localization [7] Localization suppresses both the mean current and the fluctuations-on top of the suppression due to absorption Localization is readily observed in a waveguide geometry [8], where it sets in once the length $L$ of the waveguide becomes longer than the localization length $\xi \approx N l$ (with $l$ the mean free path and $N$ the number of propagating modes) Typically, $\xi$ is much larger than the absorption length $\xi_{a}$, so that localization and absorption coexist The interplay of absorption and localızation has been studied previously for the mean current [9-12] Here we go beyond these studies to include the current fluctuations

It is instructive to contrast the super-Poissonian photonic noise with the sub-Porssonian electronic analogue In the case of electrical conduction through a disoidered wire, the (zero-temperature) noise power is smaller than the Poisson value as a result of Fermi-Dirac statistics The reduction is a factor $1 / 3$ in the absence of localization $[13,14]$ The effect of localization is to restore Poisson statistics, so that the Fano factor increases from $1 / 3$ to 1 when $L$ becomes larger than $\xi$ What we will show in this paper is that the photonic excess noise responds entirely differently to localızation Although localization suppresses $P$ and $\bar{I}$, the Fano factor remains unaffected, equal to the value $1+\frac{3}{2} f$ obtained in the absence of localization $[15,16]$

Let us begin our analysis with a more precise formulation of the problem The norse power

$$
P=\int_{-\infty}^{\infty} d t \overline{\delta I(0) \delta I(t)}
$$

quantifies the size of the time-dependent fluctuations of the photon current $I(t)=\bar{I}+\delta I(t)$ (The overbar indicates an average over many measurements on the same system ) For a Poisson process, the power $P_{\text {Posson }}=\bar{I}$ equals the mean current and the Fano factor $\mathcal{F}=P / P_{\text {Porsson }}$ equals unity We consider monochromatic radiation (frequency $\omega_{0}$ ) incident in a single mode (labeled $m_{0}$ ) on a waveguide containing a disordered medium (at temperature $T$ ) (See F1g 1) The inc1dent radiation has Fano factor $\mathcal{F}_{\text {in }}$ We wish to know how the Fano factor changes as the radiation piopagates through the waveguide

Starting point of our investigation is a formula that relates the Fano factor to the scattering matrix of the medium [15],

$$
\begin{aligned}
\mathcal{F}=1+\left[t^{\dagger} t\right]_{m_{0} m_{0}}\left(\mathcal{F}_{\mathrm{ln}}-1\right) \\
+2 f\left(\omega_{0}, T\right) \frac{\left[t^{\dagger}\left(1-\boldsymbol{r}^{\dagger}-t t^{\dagger}\right) t\right]_{m_{0} m_{0}}}{\left[t^{\dagger} t\right]_{m_{0} m_{0}}}
\end{aligned}
$$

(We have assumed detection with quantum efficiency 1 in a nariow frequency interval around $\omega_{0}$ ) The function $f(\omega, T)=[\exp (\hbar \omega / k T)-1]^{-1}$ is the Bose-Einstein function The transmission matrix $t$ and the reflection matrix $r$ are $N$ $\times N$ matrices, with $N$ the number of propagating modes at frequency $\omega_{0}$ The term proportional to $f$ in $\mathrm{Eq}$ (2) is the excess noise For a unitary scattering matrix, $r r^{\dagger}+t t^{\dagger}$ equals the unit matrix $\mathbb{1}$, hence the excess noise vanishes

In what follows we will assume that the incident radiation is in a coherent state, so that $\mathcal{F}_{\text {in }}=1$ and the deviation of $\mathcal{F}$ from unity is entirely due to the excess noise Since the Bose-Einstein function at room temperature is negligibly small at optical frequencies, one would need to use the coherent radiation from an infrared or microwave laser Alter-

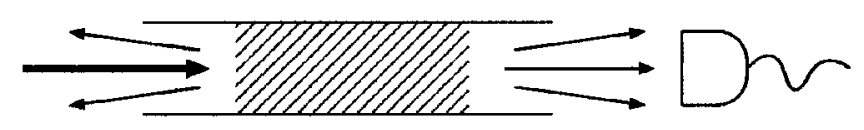

FIG 1 Monochromatic radiation (thick arrow) is incident on a disordered absorbing medium (shaded), embedded in a waveguide The transmitted radiation is measured by a photodetector 
natively, one could use a noncoherent source and extract the excess noise contubution by subtracting the noise at low temperature from that at room temperature

The absorbing disordered waveguide is characteized by four length scales the wavelength $\lambda$, the mean fiee path for scattering $l$, the absorption length $\xi_{a}$, and the localization length $\xi=(N+1) l$ We assume the ordering of length scales $\lambda \ll l \ll \xi_{a} \ll \xi$, which is the usual situation [8] We ask for the average $\langle\mathcal{F}\rangle$ of the Fano factor, averaged over an ensemble of waveguides with different realizations of the disoider For $L \gg \xi_{a}$ we may neglect the matrix $t t^{\dagger}$ with respect to $\mathbb{1}$ in Eq (2), so that the expression for the Fano factor (with $\mathcal{F}_{\text {in }}$ =1) takes the form

$$
\mathcal{F}=1+2 f\left(1-C_{1}\right), \quad C_{p} \equiv \frac{\left[t^{\dagger}\left(r r^{\dagger}\right)^{p} t\right]_{m_{0} m_{0}}}{\left[t^{\dagger} t\right]_{m_{0} m_{0}}}
$$

In the absence of localization, for $L \ll \xi$, one can simplify the calculation of $\langle\mathcal{F}\rangle$ by averaging separ ately the numerator and denominator in the coefficient $C_{1}$, since the sample-tosample fluctuations are small This diffusive regime was studied in Refs $[15,16]$ Such a simplification is no longer possible in the localized regime and we should proceed differently

We follow the general approach of Ref [12], by considering the change in $\mathcal{F}$ upon attaching a short segment of length $\delta L$ to one end of the waveguide Transmission and reflection matrices are changed to leading order in $\delta L$ accordıng to

$$
t \rightarrow t_{\delta L}\left(1+r r_{\delta L}\right) t, \quad r \rightarrow r_{\delta L}^{\prime}+t_{\delta L}\left(1+r r_{\delta L}\right) r t_{\delta L}^{T},
$$

where the supeiscript $T$ indicates the transpose of a matrix (Because of reciprocity the tiansmission matrix from left to right equals the transpose of the transmission matix from right to left ) The transmission matrix $t_{\delta L}$ of the short segment may be chosen proportional to the unit matrix,

$$
t_{\delta L}=\left(1-\delta L / 2 l-\delta L / 2 l_{\mathrm{a}}\right) \mathbb{1},
$$

where $l_{a}=2 \xi_{a}^{2} / l$ is the ballistic absorption length Unitarity of the scatteing matrix then dictates that the reflection matrix from the left of the shoit segment be related to the reflection matux from the right by $r_{\delta L}^{\prime}=-r_{\delta L}^{\dagger}$ The reflection matrix $r_{\delta L}$ is symmetric (because of reciprocity), with zero mean and variance

$$
\left\langle\left[r_{\delta L}\right]_{k l}\left[r_{\delta L}\right]_{m n}^{*}\right\rangle=\left(\delta_{k m} \delta_{l n}+\delta_{k n} \delta_{l m}\right) \delta L / \xi
$$

Substituting Eq (4) into Eq (3) and averaging we find the evolution equation

$$
\begin{aligned}
\xi \frac{d\left\langle C_{1}\right\rangle}{d L}= & -2\left\langle C_{1} \rho_{1}\right\rangle+\left\langle\rho_{2}\right\rangle \\
& -\frac{\xi l}{\xi_{a}^{2}}\left\langle C_{1}\right\rangle+1+2\left\langle C_{2}-C_{1}\right\rangle-\left\langle C_{1}^{2}\right\rangle \\
& -4 \operatorname{Re}\left\langle\left[t^{\dagger} t\right]_{m_{0} m_{0}}^{-2}\left[t^{\dagger} r t^{*}\right]_{m_{0} m_{0}}\left[t^{\mathrm{T}} r^{\dagger} r r^{\dagger} t\right]_{m_{0} m_{0}}\right\rangle
\end{aligned}
$$

$$
+2\left\langle\left(1+C_{1}\right)\left[t^{\dagger} t\right]_{m_{0} m_{0}}^{-2}\left|\left[t^{\dagger} r t^{*}\right]_{m_{0} m_{0}}\right|^{2}\right\rangle
$$

where we have defined $\rho_{p}=\mathrm{t} 1\left(1-r r^{\dagger}\right)^{p}$

Fol $L \gg \xi_{a}$ we may replace the average of the product $\left\langle C_{1} \rho_{1}\right\rangle$ by the product of averages $\left\langle C_{1}\right\rangle\left\langle\rho_{1}\right\rangle$, because [12] statistical correlations with traces that involve ieflection matrices only are of telative oider $\xi_{a} / \xi-$ which we have assumed to be $\ll 1$ The moments of the reflection matrix ale given for $L \gg \xi_{a}$ by [17]

$$
\left\langle\rho_{p}\right\rangle=\frac{\Gamma(p-1 / 2)}{\sqrt{\pi} \Gamma(p)} \frac{\xi}{\xi_{a}},
$$

hence they are $\gg 1$ and also $\gg \xi l / \xi_{a}^{2}$ We may therefore neglect the terms in the second, thind, and fourth lines of Eq (7) What remains is the differential equation

$$
\xi \frac{d\left\langle C_{1}\right\rangle}{d L}=-2\left\langle C_{1}\right\rangle\left\langle\rho_{1}\right\rangle+\left\langle\rho_{2}\right\rangle
$$

which for $L \gg \xi_{a}$ has the solution

$$
\left\langle C_{1}\right\rangle=\frac{\left\langle\rho_{2}\right\rangle}{2\left\langle\rho_{1}\right\rangle}=\frac{1}{4}
$$

We conclude that the average Fano factor $\langle\mathcal{F}\rangle=1+2 f(1$ $\left.-\left\langle C_{1}\right\rangle\right) \rightarrow 1+\frac{3}{2} f$ for $L \gg \xi_{a}$, regardless of whether $L$ is small or large compared to $\xi$

To support this analytical calculation we have carried out numerical simulations The absorbing disordered waveguide is modeled by a two-dimensional square lattice (lattice constant a) The dielectric constant $\varepsilon$ has a real part that fluctuates fiom site to site and a nonfluctuatıng imaginary part The multiple scattering of a scalar wave $\Psi$ s described by discretizing the Helmholtz equation $\left[\nabla^{2}+\left(\omega_{0} / c\right)^{2} \varepsilon\right] \Psi=0$ and computing the transmission and reflection matrices using the recursive Green-function technique [18] The mean free path $l=20 a$ and the absorption length $\xi_{a}=135 a$ are determined from the average transmission probability $N^{-1}\left\langle\operatorname{tr} t t^{\dagger}\right\rangle=l / \xi_{\mathrm{a}} \sinh \left(L / \xi_{\mathrm{d}}\right)$ in the diffusive regime [12] Averages were performed over the $N / 2$ modes $m_{0}$ near normal incidence and ovet some $10^{2}-10^{3}$ realizations of the disolder Results are shown in Figs 2 and 3

The length dependence of the average Fano factor is plotted in Fig 2, for $N=50$ and $L$ ranging from 0 to $2 \xi$ Clearly, localization has no effect The limiting value of $f^{-1}\langle\mathcal{F}-1\rangle$ resulting from this simulation is slightly smaller than the value $3 / 2$ predicted by the analytical theory for $N \gg 1$ The $N$ dependence of $\langle\mathcal{F}\rangle$ in the localized regime is shown in Fig 3 A line through the data points extrapolates to the theoretical expectation $f^{-1}\langle\mathcal{F}-1\rangle \rightarrow 3 / 2$ for $N \rightarrow \infty$ Figure 3 also shows the variance of the Fano factor The variance extrapolates to 0 for $N \rightarrow \infty$, indicating that $\mathcal{F}=P / \bar{I}$ becomes selfaveraging for large $N$ This is in contrast to $P$ and $\bar{I}$ themselves, which fluctuate stiongly in the localized regime

In conclusion, we have demonstrated that localization of radiation in an absorbing disordered waveguide has no effect on the ratio of the excess noise and the mean current In the 


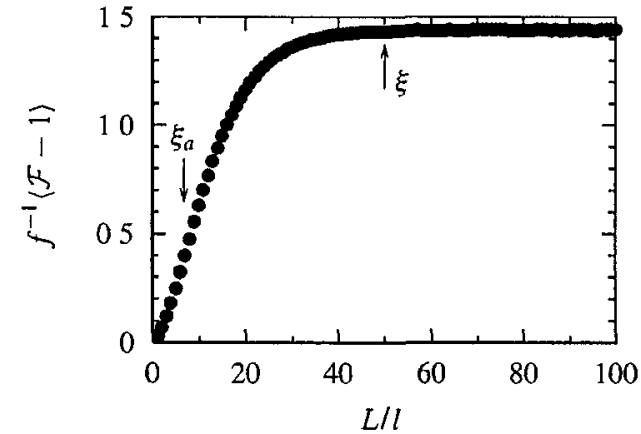

FIG 2 Length dependence of the average Fano factor, computed from Eq (2) with $\mathcal{F}_{1 \mathrm{n}}=1$ The data points result from a numerical simulation for an absorbing disordered waveguide with $N$ $=50$ propagatıng modes Arrows indicate the absorption length $\xi_{a}$ and the localization length $\xi$ The average Fano factor is not affected by localization

limit of a large number of propagating modes, this ratio is self-averaging and takes on the universal value of $3 / 2$ times the Bose-Einstem function Observation of this photonic analogue of the univeisal $1 / 3$ reduction of electronic shot noise

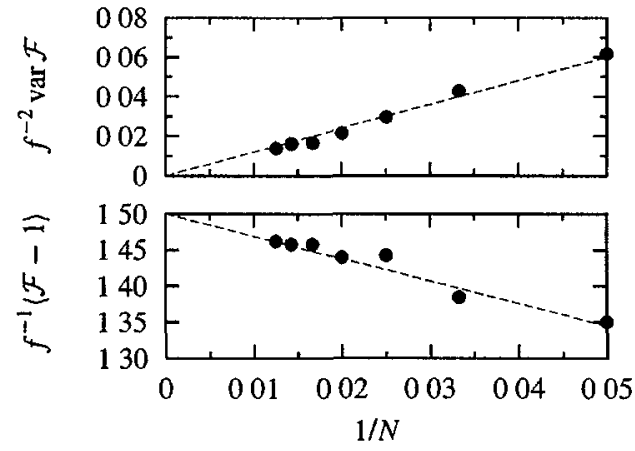

FIG 3 Dependence of the average and variance of the Fano factor on the number $N$ of propagatıng modes, for fixed length $L$ $=260 l=385 \xi_{a}$ of the waveguide The length is larger than the localization length $\xi=(N+1) l$ for all data points The dashed lines extrapolate to the theoretical expectation for $1 / N \rightarrow 0$

presents an experimental challenge

This work was supported by the Dutch Science Foundation NWO/FOM
[1] D F Walls and G J Milburn, Quantum Optıcs (Springer, Berlın, 1994)

[2] L Mandel and E Wolf, Optical Coherence and Quantum Optıcs (Cambridge Unıversity, Cambridge, 1995)

[3] C H Henry and R F Kazarınov, Rev Mod Phys 68, 801 (1996)

[4] R Matloob, R Loudon, S M Barnett, and J Jeffers, Phys Rev A 52, 4823 (1995)

[5] T Gruner and D -G Welsch, Phys Rev A 54, 1661 (1996)

[6] S M Barnett, C R Gilson, B Huttner, and N Imoto, Phys Rev Lett 77, 1739 (1996)

[7] Scattering and Localization of Classical Waves in Random Media, edited by $\mathrm{P}$ Sheng (World Scientific, Singapore, 1990)

[8] M Stoytchev and A Z Genack, Opt Lett 24, 262 (1999)

[9] P W Anderson, Philos Mag B 52, 502 (1984)
[10] R L Weaver, Phys Rev B 47, 1077 (1993)

[11] M Yosefin, Europhys Lett 25, 675 (1994)

[12] P W Brouwer, Phys Rev B 57, 10526 (1998)

[13] C W J Beenakker and M Buttıker, Phys Rev B 46, 1889 (1992)

[14] K E Nagaev, Phys Lett A 169, 103 (1992)

[15] M Patra and C W J Beenakker, Phys Rev A 60, 4059 (1999)

[16] C W J Beenakker and M Patra, Mod Phys Lett B 13, 337 (1999)

[17] These moments follow from the Laguerre distribution of the reflection etgenvalues, cf C W J Beenakker, J C J Paasschens, and P W Brouwer, Phys Rev Lett 76, 1368 (1996)

[18] H U Baranger, D P DiVincenzo, R A Jalabert, and A D Stone, Phys Rev B 44, 10637 (1991) 